\title{
STAT3 Inhibitor WP1066
}

National Cancer Institute

\section{Source}

National Cancer Institute. STAT3 Inhibitor WP1066. NCI Thesaurus. Code C111042.

An orally bioavailable, small molecule inhibitor of signaling transducer and activator 3

(STAT3), with potential antineoplastic and immunomodulatory activities. Upon

administration, STAT 3 inhibitor WP1066 blocks the intranuclear translocation of p-STAT, thereby suppressing STAT3 signaling and decreasing the levels of downstream products including c-Myc. Additionally, WP1066 may upregulate costimulatory molecules including CD80 and CD86 on human microg lia, and reverse glioma cancer stem cell (gCSC)mediated innate and adaptive immune suppression allowing for the restoration of antitumor effector immune responses. The STAT3 pathway is overly active in many cancer types and is implicated in CSC-mediated growth, recurrence and resistance to conventional chemotherapies. 